\title{
Solving the Environmental Economic Dispatch Problem with Prohibited Operating Zones in Microgrids using NSGA-II and TOPSIS
}

\author{
Marco Cococcioni, Beatrice Lazzerini, Francesco Marcelloni, Francesco Pistolesi \\ Department of Information Engineering, University of Pisa \\ Largo Lucio Lazzarino, 1 - 56122 Pisa, IT \\ \{m.cococcioni, b.lazzerini, f.marcelloni, f.pistolesi\}@iet.unipi.it
}

\begin{abstract}
This paper presents a multi-objective optimization framework for the environmental economic dispatch problem in microgrids. Besides classic constraints, also prohibited operating zones and ramp-rate limits of the generators are here considered. Pareto-optimal solutions are generated through the NSGA-II algorithm with customized constraint handling. The optimal solution is selected with TOPSIS. Simulations carried out on a prototype microgrid showed the effectiveness of the proposed framework in handling scenarios with Pareto fronts having up to four discontinuities.
\end{abstract}

\section{CCS Concepts}

- Mathematics of computing $\rightarrow$ Mathematical optimization; Bio-inspired optimization; •Hardware $\rightarrow$ Smart grid; Impact on the environment;

\section{Keywords}

Environmental economic dispatch; genetic algorithms; multiobjective optimization; smart microgrids

\section{INTRODUCTION}

Microgrids are next-generation low or medium voltage power systems integrating distributed generation units (such as photovoltaic panels, wind turbines and so on), small classic generators and energy storage systems [1]. A microgrid may be connected to the power network: in this case it works in grid-connected mode. Alternatively, a microgrid is islanded.

Energy management in a grid-connected microgrid is focused on determining the amount of energy each generation unit must produce to satisfy the current power demand at the lowest cost, given the available renewable energy: this is the economic dispatch problem (ED). In general, ED is a non-linear and non-convex problem.

Permission to make digital or hard copies of all or part of this work for personal or classroom use is granted without fee provided that copies are not made or distributed for profit or commercial advantage and that copies bear this notice and the full citation on the first page. To copy otherwise, or republish, to post on servers or to redistribute to lists, requires prior specific permission and/or a fee.

SAC 2016, April 04-08, 2016, Pisa, Italy

Copyright 2016 ACM 978-1-4503-3739-7/16/04 . .\$15.00

http://dx.doi.org/10.1145/2851613.2853130
Recently, ED has evolved to minimize both cost and environmental impact: this problem is known as environmental economic dispatch (EED). EED is a multi-objective problem. ED and EED are conventionally dealt with by assuming the power output of each unit to be continuously modifiable between its minimum and maximum limits. However, generation units may have physical limitations in certain operating regions, the so-called prohibited operating zones (POZs). The operating region of a unit with POZs is broken into several disjointed sub-regions. EED can be made more realistic (as well as more complex) by taking the ramprate limit of the generators into account, i.e., the maximum speed in changing the output power. Due to the intrinsic complexity of EED, evolutionary techniques have been recently proposed: chaotic ant swarm optimization [2], fuzzy multi-objective optimization combined with particle swarm optimization [9] and artificial bee colony algorithms mixed with Markov chains [8] are some recent applications of evolutionary computation to EED in microgrids. Also, other recent techniques try to optimize more than two objectives $[5,7,6]$. Finally, in [11] two well-known multi-objective evolutionary algorithms (SPEA2 and NSGA-II) are applied.

In this paper, the work described in [11] is made harder and more realistic by taking POZs and ramp-rate limits into account. Also, a battery is included to store the excess of energy. First, the Pareto front is here generated by means of NSGA-II. Then, an integrated TOPSIS-based multi-criteria decision making module automatically selects the best solution. The optimization framework was tested on two scenarios simulated on a prototype microgrid.

The rest of the paper is structured as follows: Section 2 gives some preliminaries of TOPSIS and NSGA-II; Section 3 contains the problem formulation; in Section 4 the optimization results are discussed; Section 5 draws the conclusions.

\section{PRELIMINARIES}

\subsection{Technique for Order of Preference by Sim- ilarity to Ideal Solution (TOPSIS)}

TOPSIS is a multi-criteria decision making technique [3]. Given a decision problem with $n$ alternatives and $m$ criteria, TOPSIS requires a decision-maker to fill an $n \times m$ decision matrix $\mathbf{H}=\left[h_{i j}\right]$, where $h_{i j}$ is the performance of alternative $i$ on criterion $j$, with $i \in\{1, \ldots, n\}$ and $j \in$ 
$\{1, \ldots, m\}$. TOPSIS also needs a vector $\boldsymbol{\omega}=\left(\omega_{1}, \ldots, \omega_{m}\right)$, where $\sum_{j=1}^{m} \omega_{j}=1$, whose elements are the weights of the criteria. To select the best alternative, TOPSIS first builds the normalized decision matrix $\mathbf{R}=\left[r_{i j}\right]=h_{i j} / \sqrt{\sum_{i=1}^{n} h_{i j}^{2}}$ and the weighted normalized decision matrix $\mathbf{V}=\left[v_{i j}\right]=$ $v_{i j}=\omega_{j} r_{i j}$. Then, the ideal best $(I B)$ and worst $(I W)$ solutions are found. Let $\Omega_{B}$ and $\Omega_{C}$ contain the indexes of benefit and cost criteria, respectively. Let $I B=\left(a_{1}^{+}, \ldots, a_{m}^{+}\right)$ and let $I W=\left(a_{1}^{-}, \ldots, a_{m}^{-}\right)$, where $a_{j}^{+}=\max _{i} v_{i j}$ for $j \in \Omega_{B}$ or $a_{j}^{+}=\min _{i} v_{i j}$ for $j \in \Omega_{C}$, and $a_{j}^{-}=\min _{i} v_{i j}$ for $j \in \Omega_{B}$ or $a_{j}^{-}=\max _{i} v_{i j}$ for $j \in \Omega_{C}$. TOPSIS measures the Euclidean distance of each alternative from $I B$, i.e., $D_{i}^{+}=$ $\sqrt{\sum_{j=1}^{m}\left(v_{i j}-a_{j}^{+}\right)^{2}}$, and $I W$, i.e., $D_{i}^{-}=\sqrt{\sum_{j=1}^{m}\left(v_{i j}-a_{j}^{-}\right)^{2}}$. Finally, TOPSIS computes the relative closeness coefficient of each alternative to $I B$ as $R C L_{i}^{+}=D_{i}^{-} /\left(D_{i}^{+}+D_{i}^{-}\right)$: the higher $R C L_{i}^{+}$the better. The best alternative is $k=$ $\arg \max _{i} R C L_{i}^{+}$.

\subsection{The NSGA-II algorithm}

NSGA-II [4] is one of the most frequently used multi-objective genetic algorithms, where the acronym means Nondominated Sorting Genetic Algorithm. From an operation point of view, NSGA-II starts to generate a population $P_{0}$ of $n$ individuals randomly, and associates each one with a non-domination rank: rank 1 for the best level, rank 2 for the next level, etc. To this aim, NSGA-II first finds the nondominated individuals in $P_{0}$ (in the sense of Pareto dominance) and assigns rank 1 to them: these individuals belong to the first front. Then, individuals of the first front are neglected to find individuals with rank 2 and so on. At iteration $t$, NSGA-II generates an offspring population $Q_{t}$ of $n$ individuals by picking from population $P_{t}$ with binary tournament selection, then applying crossover and mutation operators. $P_{t}$ and $Q_{t}$ are merged into a new population $R_{t}$. The $2 n$ individuals in $R_{t}$ are associated with their ranks so as to divide $R_{t}$ into fronts. In each front, the density of individuals in each individual's neighborhood is estimated as the sum of the distances from an individual to the closest one, along each objective (so-called crowding distance). NSGAII sorts individuals in a front according to their crowding distances. Finally, population $P_{t+1}$ is derived from $R_{t}$ discarding the worst $n$ individuals by first considering the sorting among fronts, then among individuals in the same front. NSGA-II iterates for a specified number of generations.

\section{ENVIRONMENTAL ECONOMIC DIS- PATCH IN MICROGRIDS}

\subsection{Objective functions}

Let $\mathbf{p} \in \mathbb{R}_{+}^{|\mathcal{G}|}$ be a feasible power output configuration of the generators, where $\mathcal{G}$ is the set of the generators and $|\cdot|$ denotes the cardinality of a set. The total cost function $f^{C O S T}: \mathbb{R}_{+}^{|\mathcal{G}|} \rightarrow \mathbb{R}^{+}$is $f^{C O S T}(\mathbf{p})=\sum_{i=1}^{|\mathcal{G}|} c_{i, \alpha}+c_{i, \beta} p_{i}+$ $c_{i, \gamma} p_{i}^{2}$, where $c_{i, \alpha}, c_{i, \beta}$ and $c_{i, \gamma}$ are the cost coefficients of generator $i$. Likewise, the total environmental impact is modelled through the emission function $f^{E M I S S I O N}: \mathbb{R}_{+}^{|\mathcal{G}|} \rightarrow$ $\mathbb{R}^{+}, f^{\text {EMISSION }}(\mathbf{p})=\sum_{i=1}^{|\mathcal{G}|} \pi_{i, \alpha}+\pi_{i, \beta} p_{i}+\pi_{i, \gamma} p_{i}^{2}$, where $\pi_{i, \alpha}, \pi_{i, \beta}$ and $\pi_{i, \gamma}$ are coefficients related to the emission characteristics of the $i$-th generator.

\subsection{Problem formulation}

Considered a microgrid, let $\mathcal{Z}$, where $\mathcal{Z} \subset \mathcal{G}$, be the set of the generators with POZs, and let $\mathcal{L}$ be the set of the loads. Also, let $p_{i, t}$ be the power output of the $i$-th generator at instant $t$, where $i \in\{1, \ldots,|\mathcal{G}|\}$, and let $d_{j, t}$ be the power demand of the $j$-th load at time $t$, where $j \in\{1, \ldots,|\mathcal{L}|\}$. Finally, let $\mathcal{B}$ be the set of the batteries and let $p_{k, t}$ be the charge or discharge power of battery $k$ at instant $t$, where $k \in\{1, \ldots,|\mathcal{B}|\}$. The model of the problem is the following:

$$
\text { Minimize } \mathbf{f}(\mathbf{p})=\left[f^{\operatorname{COST}}(\mathbf{p}), f^{\text {EMISSION }}(\mathbf{p})\right]
$$

subject to:

$$
\begin{gathered}
\sum_{i=1}^{|\mathcal{G}|} p_{i, t}+P_{P C C}+\sum_{k=1}^{|\mathcal{B}|} p_{k, t}=\sum_{j=1}^{|\mathcal{L}|} d_{j, t} \\
P_{i}^{\text {min }} \leq p_{i, t} \leq P_{i}^{\text {max }} \forall i \in \mathcal{G} \\
p_{i, t} \leq P_{i, 1}^{\text {min }} \vee\left(\bigvee_{z=1}^{Z_{i}-1}\left(P_{i, z}^{\text {max }} \leq p_{i, t} \leq P_{i, z+1}^{\text {min }}\right)\right) \\
\vee p_{i, t} \geq P_{i, Z_{i}}^{\text {max }} \forall i \in \mathcal{Z} \\
\left|p_{i, t-1}-p_{i, t}\right| \leq r_{i}^{\text {max }} \Delta t \forall i \in \mathcal{G} \\
-P_{k}^{\text {max charge }} \leq p_{k, t} \leq P_{k}^{\text {max discharge }} \forall k \in \mathcal{B} \\
E_{k}^{\text {min }} \leq E_{k, t-1}+p_{k, t} \Delta t \leq E_{k}^{\text {max }} \forall k \in \mathcal{B}
\end{gathered}
$$

Equation (1a) is the vector-valued objective function. Equation (1b) is the power balance of the microgrid, where $P_{P C C}$ is the power exchanged with the main grid at the point of common coupling. Power losses are neglected because of the small scale of the microgrid. Equation (1c) represents the power limits of the generators, where $P_{i}^{\text {min }}$ and $P_{i}^{\text {max }}$ are, respectively, the minimum and maximum power of the $i$-th generator. Equation (1d) is a disjunctive constraint defining the feasible operating zones of the generators in $\mathcal{Z}, Z_{i}$ is the number of POZs of generator $i$, and $P_{i, z}^{\min }$ and $P_{i, z}^{\max }$ are, respectively, the minimum and the maximum power of the $z$-th POZ of generator $i$. Equation (1e) defines the ramp constraint, one for each generator, where $r_{i}^{\max }$ is the ramprate limit of generator $i$ and $\Delta t$ is the time period from an optimization to the next one. The last two constraints are related to the battery storage. In particular, Equation (1f) limits the charge and discharge power; $P_{k}^{\text {max charge }}$ and $P_{k}^{\text {max discharge }}$ are, respectively, the maximum charge and the maximum discharge power of battery $k$. Equation (1g) is the energy storage constraint, where, for each battery $k$, $E_{k}^{\min }$ and $E_{k}^{\max }$ are the minimum and maximum energy level, $E_{k, t-1}$ is the energy level at instant $t-1$.

\section{OPTIMIZATION RESULTS}

The proposed optimization framework was implemented in MATLAB. Simulations were carried out on a workstation equipped with an Intel $^{\circledR}$ i5 $3.0 \mathrm{GHz}$ processor and $8 \mathrm{~GB}$ of RAM, by considering a prototype microgrid composed of two thermal generators (TH1 and TH2), two diesel engines (DE1 and DE2), a fuel cell (FC), a photovoltaic panel (PV), 
a wind turbine (WT) and a battery (BS). Power limits and ramp-rate limits of the generators are summarized in Table 1, while cost and emission coefficients are in Table 2. BS is assumed to have $P_{k}^{\text {max discharge }}=30 \mathrm{~kW}$ and $P_{k}^{\text {max charge }}=$ $-P_{k}^{\text {max discharge }}$. The microgrid is also assumed in gridconnected mode. NSGA-II was set up with a population

Table 1: Power limits and ramp-rate limits of the generators

\begin{tabular}{ccccc}
\hline \multirow{2}{*}{ ID } & Type & \multicolumn{2}{c}{$\begin{array}{c}\text { Power } \\
(\mathbf{k W})\end{array}$} & $\begin{array}{c}\text { Ramp-rate } \\
(\mathbf{k W} / \mathbf{m i n})\end{array}$ \\
\cline { 3 - 5 } & & $\boldsymbol{P}_{\boldsymbol{i}}^{\text {min }}$ & $\boldsymbol{P}_{\boldsymbol{i}}^{\text {max }}$ & $\boldsymbol{r}_{\boldsymbol{i}}^{\text {max }}$ \\
\hline TH1 & Thermal & 5 & 950 & 1.5 \\
TH2 & Thermal & 5 & 950 & 1.5 \\
DE1 & Diesel engine & 0 & 30 & 1 \\
DE2 & Diesel engine & 10 & 35 & 0.75 \\
FC & Fuel cell & 0 & 25 & - \\
PV & Photovoltaic panel & 0 & 10 & - \\
WT & Wind turbine & 0 & 40 & - \\
\hline
\end{tabular}

Table 2: Cost and emission coefficients of the generators

\begin{tabular}{ccccccc}
\hline \multirow{2}{*}{ ID } & \multicolumn{2}{c}{ Cost coefficients } & \multicolumn{3}{c}{ Emission coefficients } \\
\cline { 2 - 7 } & $\boldsymbol{c}_{\boldsymbol{i}, \boldsymbol{\alpha}}$ & $\boldsymbol{c}_{\boldsymbol{i}, \boldsymbol{\beta}}$ & $\boldsymbol{c}_{\boldsymbol{i}, \boldsymbol{\gamma}}$ & $\boldsymbol{\pi}_{\boldsymbol{i}, \boldsymbol{\alpha}}$ & $\boldsymbol{\pi}_{\boldsymbol{i}, \boldsymbol{\beta}}$ & $\boldsymbol{\pi}_{\boldsymbol{i}, \boldsymbol{\gamma}}$ \\
\hline TH1 & 10 & 100 & 60 & $4.091 \mathrm{e}-2$ & $-5.554 \mathrm{e}-2$ & $6.490 \mathrm{e}-2$ \\
TH2 & 10 & 100 & 60 & $4.091 \mathrm{e}-2$ & $-5.554 \mathrm{e}-2$ & $6.490 \mathrm{e}-2$ \\
DE1 & 10 & 200 & 100 & $5.326 \mathrm{e}-2$ & $-3.550 \mathrm{e}-2$ & $3.380 \mathrm{e}-2$ \\
DE2 & 15 & 250 & 110 & $5.186 \mathrm{e}-2$ & $-3.799 \mathrm{e}-2$ & $4.654 \mathrm{e}-2$ \\
FC & 20 & 180 & 40 & $4.846 \mathrm{e}-2$ & $-4.465 \mathrm{e}-2$ & $3.994 \mathrm{e}-2$ \\
PV & - & 50 & - & - & - & - \\
WT & - & 25 & - & - & - & - \\
\hline
\end{tabular}

of 100 individuals. Each individual is composed of seven real-coded genes, and the gene in position $i$ represents the power output of the $i$-th generator, where $i \in\{1, \ldots, 7\}$. In practice, gene in position 1 is associated with $\mathrm{TH} 1$, gene in position 2 with TH2 and so on. The chosen maximum number of generations was 2000. Simulated binary crossover [10] was applied with a probability of 0.9 ; if not applied, polynomial mutation [10] was performed. Candidate parents were randomly selected.

Constraint handling was performed with a two-fold approach. Constraints on power limits, POZs and ramp-rate limits, in Eqs. (1c), (1d) and (1e), respectively, were handled according to a repairing technique. In particular, once an offspring is generated, if some of its genes fall into a POZ or exceed a power limit or a ramp-rate limit, randomly mutation is performed until constraints are met. The obtained solutions might be infeasible due to violations of the power balance constraint in Eq. (1b). For the power balance constraint handling, a penalty method was chosen. More in detail, both objectives were penalized by a non-negative penalty term $\gamma(\mathbf{p})$, weighted using two coefficients $\omega_{C}, \omega_{E} \in \mathbb{R}^{+}$, so as to obtain the following functions: $g^{C O S T}(\mathbf{p})=f^{C O S T}(\mathbf{p})+$ $\omega_{C} \gamma(\mathbf{p}) ; g^{E M I S S I O N}(\mathbf{p})=f^{E M I S S I O N}(\mathbf{p})+\omega_{E} \gamma(\mathbf{p})$. Let $\Delta_{t}^{P O W E R}=\sum_{i=1}^{|\mathcal{G}|} p_{i, t}+P_{P C C}+\sum_{k=1}^{|\mathcal{B}|} p_{k, t}-\sum_{j=1}^{|\mathcal{L}|} d_{j, t}$. The penalty term $\gamma(\mathbf{p})$ has the following expression:

$$
\gamma(\mathbf{p})= \begin{cases}\xi_{1} \Delta_{t}^{P O W E R} & \text { if } \Delta_{t}^{P O W E R} \geq 0 \\ \xi_{2}\left|\Delta_{t}^{P O W E R}\right| & \text { otherwise }\end{cases}
$$

where $\xi_{1}, \xi_{2} \in \mathbb{R}^{+}$. To achieve a performance-effective constraint handling, values of $\omega_{C}, \omega_{E}, \xi_{1}$ and $\xi_{2}$ were determined experimentally by performing 30 executions of NSGAII. At the end of each execution, the overall average constraint violation was measured, and parameters were appropriately changed. Final values were: $\omega_{C}=1000 ; \omega_{E}=1$; $\xi_{1}=100 ; \xi_{2}=1$.

Optimization results are presented for two scenarios (Scenario A and Scenario B) in the following subsections.

\subsection{Scenario A}

This scenario assumes a $150 \mathrm{~kW}$ power demand, which may be a typical off-peak load of a small residential area. The considered time period was $\Delta t=10 \mathrm{~min}$. $6 \mathrm{~kW}$ and $30 \mathrm{~kW}$ power productions were assumed for PV and WT, respectively. Also, the power dispatch at instant $t-1$ was sup-

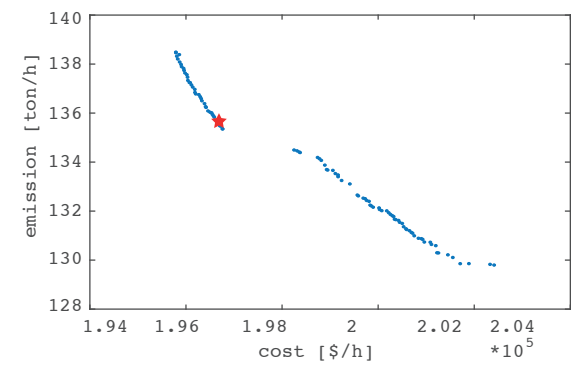

Figure 1: Final Pareto front of Scenario A

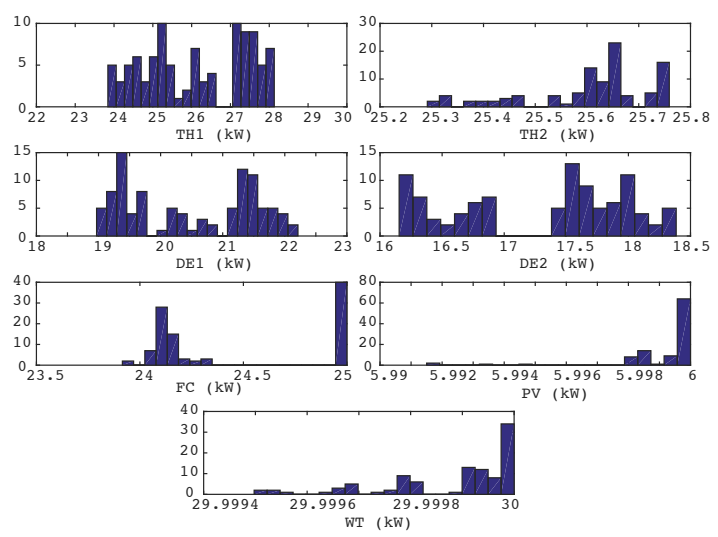

Figure 2: Distribution of the individuals' genes in the final population of Scenario A

posed to be $15 \mathrm{~kW}$ for TH1, $22 \mathrm{~kW}$ for TH2, $14 \mathrm{~kW}$ for DE1 and $13 \mathrm{~kW}$ for DE2. POZs are $[50 \mathrm{~kW}, 75 \mathrm{~kW}]$ for TH1 and $[35 \mathrm{~kW}, 80 \mathrm{~kW}]$ for TH2. The Pareto front approximation, shown in Fig. 1, was obtained in 2 minutes and 16 seconds. As it can be seen, the central gap makes the front noncontinuous. In particular, constraints here generate two distinct sub-regions. The best solution (depicted with a star mark) is automatically selected by TOPSIS with weights 0.3 and 0.7 , for, respectively, cost and emissions. To choose such weights, we imagined a scenario wherein emissions play a quite critical role, thus associating the corresponding objective function with an importance 2.3 times higher than the one associated with cost. The distribution of the individuals' genes in the final population is shown in Fig. 2. It can be seen that POZs are avoided. Also, it can be easily 
verified how the ramp-rate limit constraints are met by all the individuals. Finally, it is interesting to note that renewable energy sources contribute to satisfy the load demand at almost their maximum power $(6 \mathrm{~kW}$ and $30 \mathrm{~kW}$, respectively). As one might expect, this is likely related to the high importance here given to emissions.

\subsection{Scenario B}

With this scenario we investigated a harder situation than Scenario A. To this aim, a further POZ is here introduced for generator TH1 in the power range $[20 \mathrm{~kW}, 30 \mathrm{~kW}]$. The obtained Pareto front is shown in Fig. 3, which also provides the solution selected by TOPSIS using the same weights as Scenario A. As we can see by comparing Fig. 3 to Fig. 1,

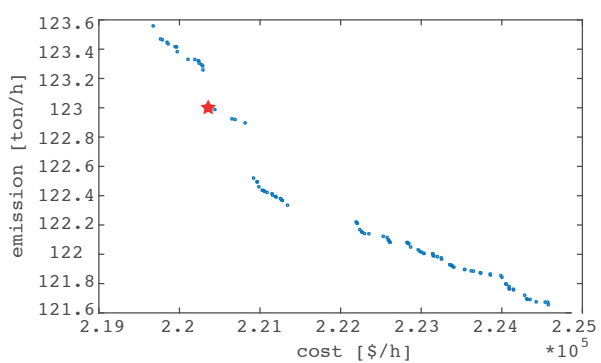

Figure 3: Final Pareto front of Scenario B

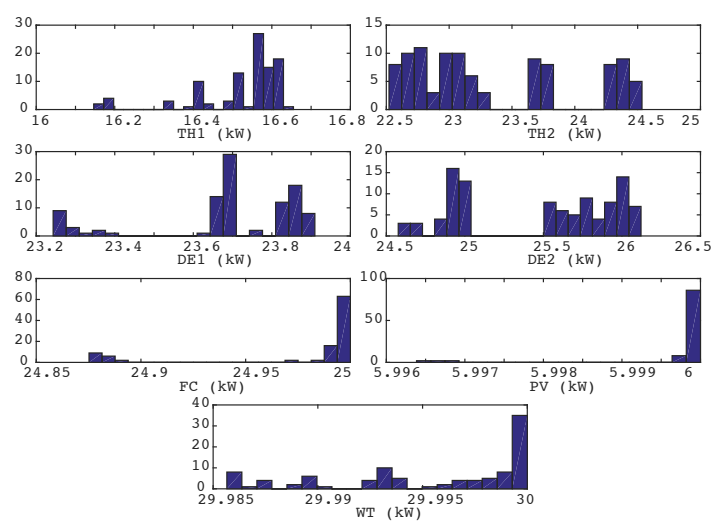

Figure 4: Distribution of the individuals' genes in the final population of Scenario $B$

the further POZ caused a considerably different shape and a higher fragmentation of the Pareto front: four sub-regions are clearly highlighted in this scenario. Increased fragmentation is due to the further separation of the feasible region in the search space, generated by the new POZ. Nevertheless, performance did not show a significant reduction. The optimal solution was found in 2 minutes and 38 seconds, with an increase of $\sim 16.18 \%$ of the execution time if compared to Scenario A. Fig. 4 shows the distribution of the individuals' genes in the final population. Even in this case, renewable energy sources were fully used to satisfy the load. Due to the additional POZ in the range $[20 \mathrm{~kW}, 30 \mathrm{~kW}]$ for TH1, the optimal power output of TH1 in Scenario A (approximately $25 \mathrm{~kW}$ ) is no longer feasible: a value around and below $16 \mathrm{~kW}$ was selected instead. Finally, a comparison between the distributions of the individuals' genes in the simulated scenarios (see Fig. 2 and Fig. 4) clearly shows that, on average, here DE2 must provide a much higher power to satisfy the load.

\section{CONCLUSIONS}

This paper presented an evolutionary computation-based approach for solving the EED problem with POZs and ramprate limits in a microgrid. The Pareto front is approximated with NSGA-II and the best solution is selected with TOPSIS. Two scenarios were simulated on a prototype microgrid. The proposed framework proved to effectively deal with POZs and ramp-rate limits constraints, efficiently managing scenarios with up to four discontinuities.

\section{ACKNOWLEDGMENTS}

This work is partially supported by the project "Metodologie e Tecnologie per lo Sviluppo di Servizi Informatici Innovativi per le Smart Cities", funded by "Progetti di Ricerca di Ateneo - PRA 2015" of the University of Pisa.

\section{REFERENCES}

[1] S.-J. Ahn et al. Power scheduling of distributed generators for economic and stable operation of a microgrid. IEEE Transactions on Smart Grid, 4(1):398-405, 2013.

[2] J. Cai et al. A multi-objective chaotic ant swarm optimization for environmental/economic dispatch. International Journal of Electrical Power \& Energy Systems, 32(5):337-344, 2010.

[3] K. Y. Ching-Lai Hwang. Multiple attribute decision making. Springer-Verlag, 1981.

[4] K. Deb et al. A fast and elitist multiobjective genetic algorithm: NSGA-II. IEEE Transactions on Evolutionary Computation, 6(2):182-197, 2002.

[5] B. Lazzerini and F. Pistolesi. Efficient energy dispatching in smart microgrids using an integration of fuzzy AHP and TOPSIS assisted by linear programming. In Conference of the European Society for Fuzzy Logic and Technology, pages 310-317, 2013.

[6] B. Lazzerini and F. Pistolesi. Neural network-based objectives prioritization for multi-objective economic dispatch in microgrids. In IEEE/SICE International Symposium on System Integration, pages 665-671, Tokyo, JP, 2014.

[7] B. Lazzerini and F. Pistolesi. A linear programming driven MCDM approach for multi-objective economic dispatch in smart grids. In IEEE/SAI Intelligent Systems Conference, pages 475-484, London, UK, 2015.

[8] M. Marzband et al. An optimal energy management system for islanded microgrids based on multiperiod artificial bee colony combined with Markov chain. IEEE Systems Journal, PP(99):1-11, 2015.

[9] M. Meiqin et al. Multi-objective economic dispatch model for a microgrid considering reliability. In IEEE International Symposium on Power Electronics for Distributed Generation Systems, pages 993-998, 2010.

[10] M. M. Raghuwanshi and O. G. Kakde. Survey on multiobjective evolutionary and real coded genetic algorithms. In Asia Pacific Symposium on Intelligent and Evolutionary Systems, pages 150-161, 2004.

[11] J. Xu et al. Optimization of economic load dispatch for a microgrid using evolutionary computation. In Annual Conference of the IEEE Industrial Electronics Society, pages 3192-3197, 2011. 